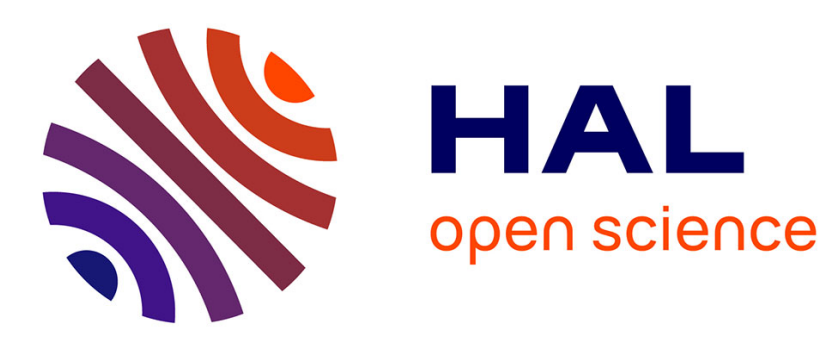

\title{
Performances intrinsèques des machines à réluctance variable à disques imbriqués
}

\author{
J.P. Bastos, R. Goyet, J. Lucidarme
}

\section{To cite this version:}

J.P. Bastos, R. Goyet, J. Lucidarme. Performances intrinsèques des machines à réluctance variable à disques imbriqués. Revue de Physique Appliquée, 1980, 15 (1), pp.45-54. 10.1051/rphysap:0198000150104500 . jpa-00244697

\section{HAL Id: jpa-00244697 https://hal.science/jpa-00244697}

Submitted on 1 Jan 1980

HAL is a multi-disciplinary open access archive for the deposit and dissemination of scientific research documents, whether they are published or not. The documents may come from teaching and research institutions in France or abroad, or from public or private research centers.
L'archive ouverte pluridisciplinaire HAL, est destinée au dépôt et à la diffusion de documents scientifiques de niveau recherche, publiés ou non, émanant des établissements d'enseignement et de recherche français ou étrangers, des laboratoires publics ou privés. 


\title{
Performances intrinsèques des machines à réluctance variable à disques imbriqués
}

\author{
J. P. Bastos, R. Goyet et J. Lucidarme \\ Laboratoire d'Electrotechnique des Universités Paris VI et XI, \\ Bât. 214, 91405 Orsay, France
}

(Reçu le 10 juillet 1979, révisé le 2 octobre 1979, accepté le 5 octobre 1979)

\begin{abstract}
Résumé. - L'étude qui suit concerne un type particulier de machine électrotechnique à grande densité de puissance : les machines à réluctance variable à disques imbriqués et grande surface d'entrefer. Une méthode de calcul numérique par éléments finis, incluant la saturation du matériau magnétique est utilisée pour déterminer de façon précise leurs performances. L'étude est orientée vers la recherche des formes géométriques optimisant la puissance volumique. Les résultats sont présentés en fonction de paramètres réduits, dont le choix optimal est exposé en fin d'article.
\end{abstract}

\begin{abstract}
The theoretical study of a high power multi-interleaved disk variable reluctance machine is discussed. This type of machine has a large air gap surface and is potentially capable of very high power densities. A finite elements numerical method has been used to precisely calculate the performance as a function of the geometrical configuration of the magnetic material so that the machine can be optimised to have a maximum power density. The results are presented in a reduced parameter form and the choice of parameters used is discussed at the end of the article.
\end{abstract}

1. Introduction. - Une théorie générale comparative des machines faite à partir des équations de l'électromagnétisme [9] met en évidence qu'au-delà des machines classiques, d'autres types de machines peuvent être très intéressantes :

- Machines à aimants permanents pour de faibles puissances.

- Machines sans fer.

- Machines utilisant des supraconducteurs.

- Machines à réluctance variable à grandes surfaces d'entrefer.

Les quatre types de machines sont toutes susceptibles, dans leur propre domaine de performances théoriques supérieures aux machines conventionnelles. Le présent travail essaie de chiffrer les performances de celles du quatrième type, à réluctance variable.

Dans une deuxième étude [1], une théorie des machines comportant des matériaux magnétiques dégage le concept d'échelle macroscopique lié aux dimensions de bobinages et celui d'échelle microscopique lié aux dimensions des dents et encoches que l'on trouve dans la plupart des machines. Si $L$ et $l$ sont les dimensions caractéristiques de ces deux domaines, on montre pour une étude comparative approchée, que lorsque le rapport $L / l$ est suffisamment grand, il est possible de concevoir des machines de performances supérieures à tout autre type de machine conventionnelle. Cette condition est réalisée sur la structure étudiée; celle-ci, dite à disques imbriqués, est représentée sur la figure 1. On distingue deux séries de disques alternativement fixes et mobiles. L'ensemble est disposé à l'intérieur d'un bobinage inducteur,

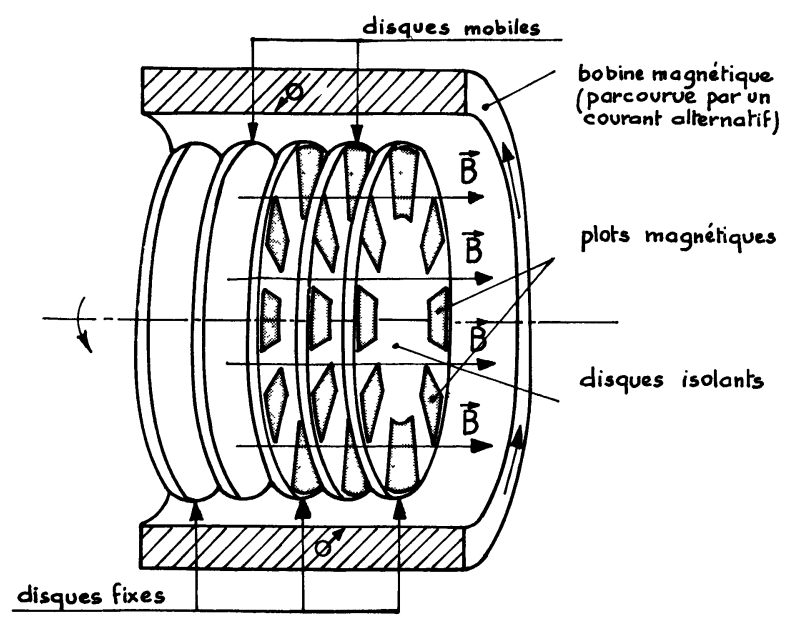

Fig. 1. - Structure d'une machine à disque imbriqués.

[Structure of an interleaved disks machine.] 
créant un champ d'induction globale B. Les disques, en matière isolante non magnétique possèdent un grand nombre de plots en matériau magnétique. Tous les plots des disques fixes sont situés en regard les uns des autres. Il en est de même pour ceux des disques mobiles.

La variation de réluctance du circuit est obtenue lors de la rotation de l'ensemble des disques mobiles. Cette réluctance est minimale lorsque tous les plots sont en face ou en conjonction; elle est maximale lorsqu'ils sont décalés ou en opposition. En fonctionnement moteur le cycle idéal est composé d'une montée rapide du courant dans la bobine en opposition, puis d'une rotation à courant constant, et enfin d'une décroissance rapide du courant en conjonction. La puissance volumique $r$ obtenue est alors le produit de l'énergie mécanique $W$ recueillie sur un cycle par la fréquence $f$ de répétition de ces cycles.

$\mathrm{La}$ réalisation de telles machines est liée aux deux problèmes particuliers suivants :

- la détermination numérique chiffrée de leurs performances, en tenant compte du régime de saturation,

- l'emploi de hautes fréquences (de l'ordre du kilohertz).

Seul le premier point est abordé dans le cadre de cet article.

Les études envisagées concernent de plus uniquement la région utile des disques, celle générant les variations de réluctance. Les performances envisagées, dites intrinsèques, font donc, abstraction des dispositifs extérieurs au cœur de la machine (bobine d'excitation, circuit magnétique de retour, sources de courants), et des pertes qui y sont engendrées.

2. Modèle numérique. -2.1 LE DOMAINE ÉLÉMENTAIRE. - La géométrie du problème est tridimensionnelle, ce qui le rend délicat à résoudre. On a donc étudié d'abord un modèle approché en négligeant la dépendance radiale. Celle-ci est abordée ensuite au paragraphe 3 . Le problème a ainsi été ramené à celui

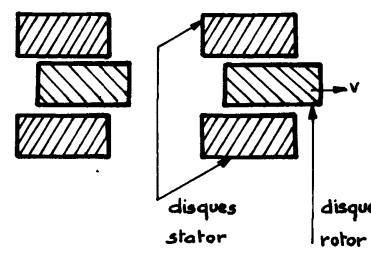

a)

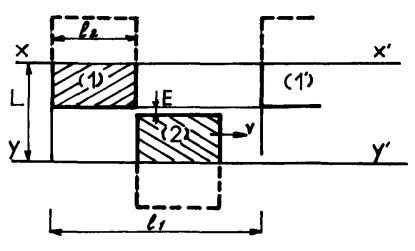

b)
Fig. 2a. - Développement bidimensionnel des surfaces d'interaction.

[Bidimensional projection of interaction surfaces.]

Fig. 2b. - Domaine élémentaire adopté sur le modèle numérique.

[Shape of the elementary domain adopted in the numerical model.] de la figure $2 a$. Par suite des conditions de périodicité, l'étude se réduit à celle de la maille élémentaire de la figure $2 b$, définissant les principales dimensions. La forme géométrique de la maille est représentée alors par les paramètres de forme suivants :

$$
e=\frac{E}{L} \quad \lambda=\frac{l_{1}}{L} \quad \text { et } \quad s=\frac{l_{2}}{l_{1}}
$$

2.2 CyCle De fonctionnement. - Le courant inducteur crée une différence de potentiel magnétique (D.D.P.) $I_{0}$ entre les lignes équipotentielles $X X^{\prime}$ et $Y Y^{\prime}$. Celle-ci engendre un flux magnétique $\Phi$ sur une largeur $l_{1}$ et sur une profondeur unité. Lorsque les ampères tours varient en fonction de l'angle de rotation $\theta$ suivant une allure de crénaux (Fig. $3 a$ ), le cycle de fonctionnement représenté sur la figure $3 b$ met en évidence, dans le plan $\Phi, I$, la surface hachurée

$$
W=\int_{\text {cycle }} I \mathrm{~d} \Phi,
$$

représentant l'énergie recueillie sur un cycle dans un volume $l_{1} \times L \times 1$.

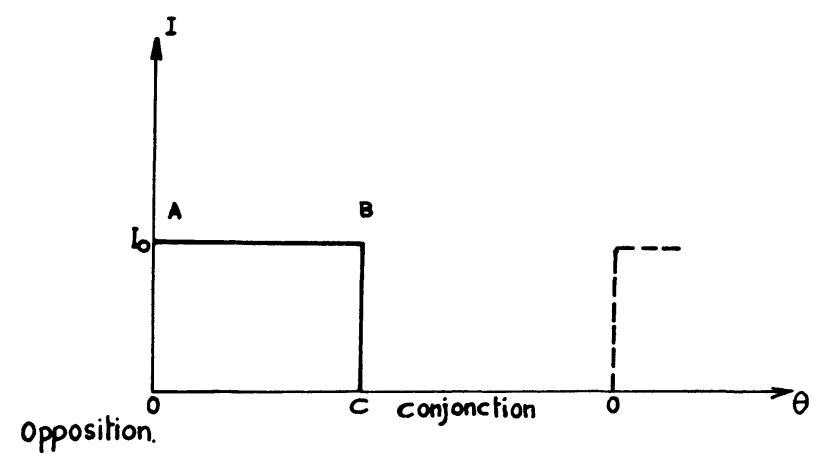

Fig. 3a. - Variation des ampères tours inducteurs.

[Field ampere turn variation.]

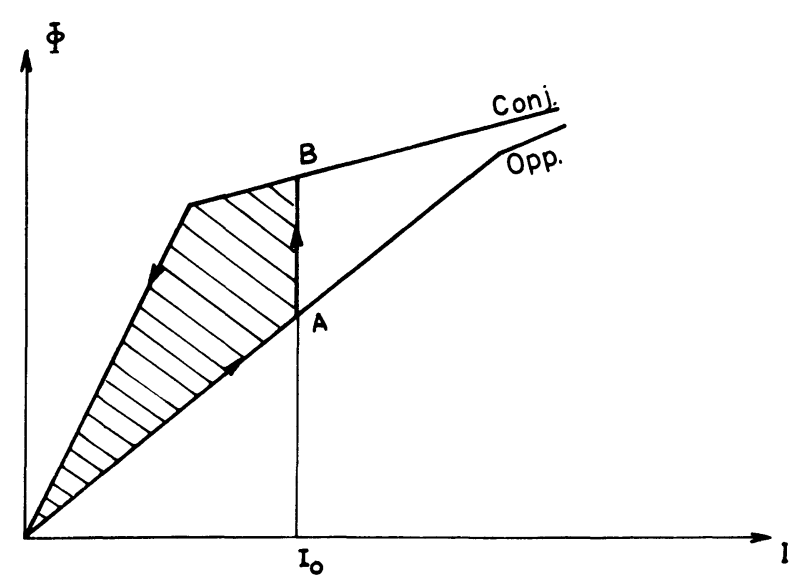

Fig. $3 b .-$ Cycle de fonctionnement dans le plan flux-ampère tours.

[Work cycle in the flux-ampere turn plan.] 
2.3 DesCRIPTION SUCCINCTE DU PROGRAMME DE CALCUL. - Les calculs de flux nécessaires à l'obtention des performances de la machine ne peuvent être déterminés que par des méthodes numériques. Le programme adopté permet de travailler sur des conditions très larges de saturation. Il utilise une méthode variationnelle par éléments finis adaptée aux problèmes de calcul de champ [2], [3], [4].

Les données principales fournies au programme sont : la D.D.P. magnétique entre $X X^{\prime}$ et $Y Y^{\prime}$ (Fig. $2 b$ ), la forme géométrique du plot et les caractéristiques du matériau magnétique.

Le programme résout les équations de la magnétostatique :

$$
\begin{gathered}
\text { Rot } \mathbf{H}=0 ; \quad \operatorname{div} \mathbf{B}=0 \text { avec } B=\mu(|H|) \cdot H \\
\mathbf{H}=-\operatorname{grad} V=-\nabla V
\end{gathered}
$$

$V$ étant la D.D.P. magnétique ou le potentiel scalaire dont dérive le champ $\mathbf{H}=-\nabla V$. Ceci conduit à

$$
\nabla(\mu|\nabla V|) \cdot \nabla V)=0 .
$$

Le potentiel scalaire $V$ est calculé aux nœuds du domaine par une inversion matricielle directe à l'aide de la méthode de Gauss [5].

Le programme calcule, entre d'autres résultats, les grandeurs $\mathbf{B}$ et $\mathbf{H}$ dans tout le domaine et, donc, le flux traversant celui-ci.

Toutes ces grandeurs sont en unités réduites. Les passages aux unités réelles sont indiqués au paragraphe suivant.

2.3.1 Fonction de perméabilité. - Le champ utilisé dans le calcul est $h=H / H_{\mathrm{c}}, H_{\mathrm{c}}$ étant le champ au coude de saturation et $H$ le champ réel.

On a :

$$
\begin{array}{ll}
|h| \leqslant 1 & \text { régime non saturé } \\
|h| \geqslant 1 & \text { régime saturé }
\end{array}
$$

La D.D.P. magnétique réelle $V$ se transforme en $v$. On a $\mathbf{h}=-\nabla \vartheta$. De la même façon le flux réel $\Phi$ est représenté par un flux réduit $\mathcal{F}$.

Pour la fonction de perméabilité relative nous avons choisi pour

$$
\begin{aligned}
& |h| \leqslant 1 \quad \mu_{\mathrm{r}}(h)=\mu(h=0) \mathrm{e}^{\frac{-G h^{3}}{3}} \\
& |h| \geqslant 1 \quad \mu_{\mathrm{r}}(h)=1+\frac{\mu(h=1)-1}{h}
\end{aligned}
$$

$\mu(h=0)$ et $G$ étant des constantes caractéristiques du matériau. Nous avons utilisé $\mu(h=0)=1000$ et $G=0,9986$.

Le champ $H=h H_{\mathrm{c}}$ donne une induction

$$
B=\mu_{0} \mu_{\mathrm{r}}(h) . h . H_{\mathrm{c}} .
$$

Lorsque $h=1$ on a $B=B_{\text {saturation }}=B_{\mathrm{s}}$

$$
B_{\mathrm{s}}=\mu_{0} \mu_{\mathrm{r}}(h=1) H_{\mathrm{c}}=\mu_{0} \mu(h=0) \mathrm{e}^{-G / 3} H_{\mathrm{c}}
$$$$
\text { REVUE DE PHYSIQUe APPLIQUÉE. - T. 15, No } 1 \text {, JANVIER } 1980
$$

soit ici :

$$
B_{\mathrm{s}}=7,169 \times 10^{2} \mu_{0} H_{\mathrm{c}} .
$$

Les résultats numériques sont indépendants de l'induction de saturation du matériau employé. $\mathrm{Si}$ le matériau utilisé est tel que $B_{\mathrm{s}}=2 \mathrm{~T}$, nous aurons $H_{\mathrm{c}} \simeq 2220 \mathrm{~A} / \mathrm{m}$. Cette valeur de $H_{\mathrm{c}}$ permet de passer des résultats numériques aux grandeurs réelles. La figure 4 montre un exemple de fonction de perméabilité pour laquelle $B_{\text {sat }}=2,2 \mathrm{~T}$, valeur couramment obtenue avec les alliages fer-cobalt.

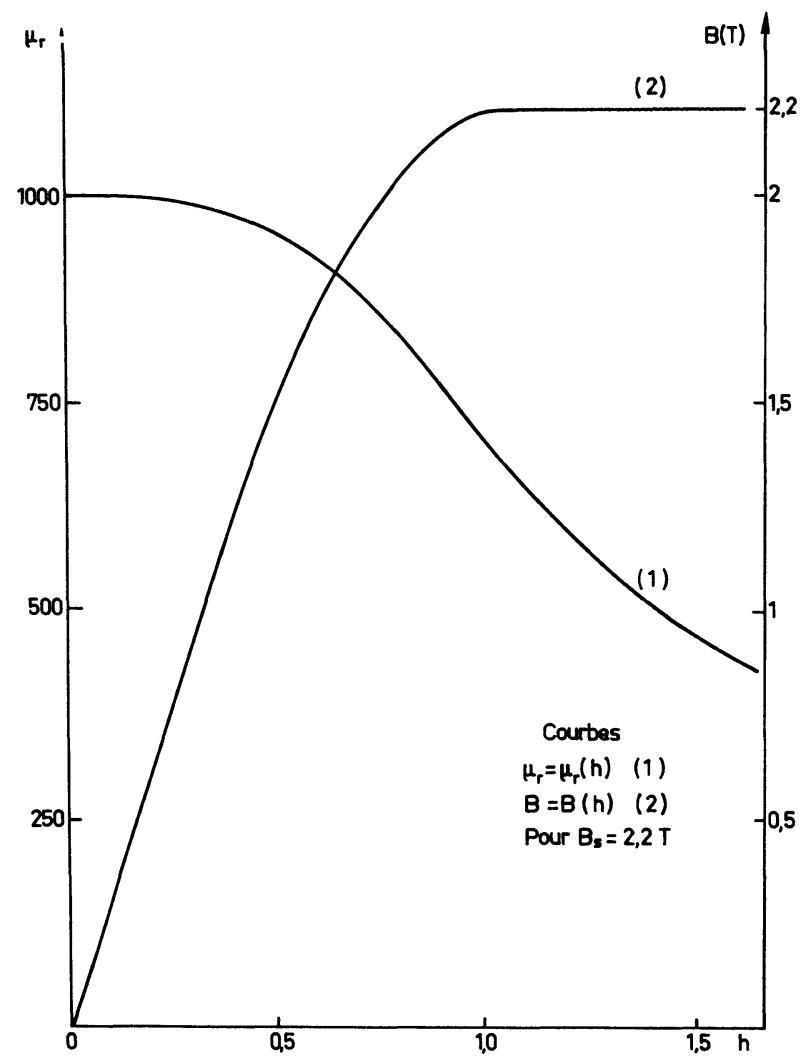

Fig. 4. - Perméabilité du matériau magnétique.

[Permeability of the magnetic material used.]

2.3.2 Courant, flux, travail par cycle et puissance volumique. - Le calcul sur ordinateur utilise le domaine de la figure $2 b$ réduit dans le rapport $L$. Les cotes $E, L, l_{1}$ et $l_{2}$ deviennent $e, 1, \lambda$ et $\lambda s$.

Nous avons donc

$$
\mathcal{V}=\int_{0}^{1} h_{y} \mathrm{~d} y \text { et } \mathfrak{F}=\int_{0}^{\lambda} \mu_{\mathrm{r}} h_{y} \mathrm{~d} x .
$$

Pour une largeur élémentaire $L$ de machine, avec le changement de variable $Y=L y, X=L x$ (où $Y$ et $X$ représentent les coordonnées réelles et $y$ et $x$ les coordonnées réduites de la maille élémentaire) nous avons :

$$
I=\int_{0}^{L} H_{Y} \mathrm{~d} Y=H_{\mathrm{c}} L \int_{0}^{1} h_{y} \mathrm{~d} y=H_{\mathrm{c}} L \mho
$$


et

$$
\Phi=\int_{0}^{1} B_{Y} \mathrm{~d} X=\mu_{0} H_{\mathrm{c}} L \int_{0}^{\lambda} \mu_{\mathrm{r}} h_{y} \mathrm{~d} x=\mu_{0} H_{\mathrm{c}} L \mathcal{F} .
$$

Les courbes $\mathcal{F}(\mathcal{U})$ fournies par l'ordinateur donnent alors directement les fonctions $\Phi(I)$ de la machine. Le travail par cycle dans un volume $l_{1} \times L \times 1$ est :

$$
W=\int_{\text {cycle }} I \mathrm{~d} \Phi=\mu_{0} H_{\mathrm{c}}^{2} L^{2} \int_{1, \lambda} \vartheta \mathrm{d} \mathcal{F} .
$$

Le travail, par unité de volume utile, est donc :

$$
W_{0}=\frac{W}{L \times l_{1} \times 1}=\frac{\mu_{0} H_{\mathrm{c}}^{2}}{\lambda} \int_{1, \lambda} \vartheta \mathrm{d} \mathcal{F} \text {. }
$$

La puissance volumique $P$ peut s'exprimer alors à l'aide de la vitesse $V$ de défilement des plots ou de la fréquence $f$ de parcours des cycles.

$$
\begin{gathered}
\text { On a }: P=W_{0} f=W_{0} \frac{V}{\lambda L} \\
P=\frac{\mu_{0} H_{\mathrm{c}}^{2}}{\lambda^{2}} \frac{V}{L} \int_{1, \lambda} \mho \mathrm{d} \mathcal{F}
\end{gathered}
$$

que nous pouvons écrire :

$$
P=\mu_{0} H_{\mathrm{c}}^{2} \frac{V}{L} A \text { avec } A=\frac{\int_{1, \lambda} \vartheta \mathrm{d} \mathcal{F}}{\lambda^{2}} .
$$

Compte tenu de la relation liant $H_{\mathrm{c}}$ et $B_{\mathrm{s}}$ nous avons :

$$
\begin{aligned}
P=3,9 \times 10^{-6}\left(\frac{B_{\mathrm{s}}^{2}}{2 \mu_{0}}\right) \times & \\
& \times \frac{V}{L} A=3,9 \times 10^{-6}\left(\frac{B_{\mathrm{s}}^{2}}{2 \mu_{0}}\right)^{\prime} \frac{R}{L} \Omega A
\end{aligned}
$$

$\Omega$ et $R$ étant respectivement la vitesse de rotation et la distance à l'axe de l'élément de volume considéré.

La dernière expression de $P$ montre que :

- L'induction de saturation du matériau doit être aussi élevée que possible.

- L'épaisseur des disques, peu différente de $L$, doit être aussi faible que possible.

- Une fois les paramètres $R$ et $\Omega$ choisis en fonction du problème à résoudre, il reste à prendre une fonction $A(e, \lambda, s, \vartheta)$ aussi élevée que possible, pour optimiser la puissance volumique.

Les résultats du calcul concernent donc la fonction $A$. Celle-ci possède une signification physique de première importance : $A$, nombre sans unité, est directement liée à la pression magnétique tangentielle moyenne $P_{\mathrm{T}}$. On recueille en effet dans un volume $\left(\lambda L, L, l_{3}\right)$ et pour un cycle de fonctionnement le travail :

$$
W=\mu_{0} H_{\mathrm{c}}^{2} L^{2} l_{3}\left(A \lambda^{2}\right)=\left[P_{\mathrm{T}}\left(\lambda L l_{3}\right)\right] \times \lambda L
$$

ce qui donne $P_{\mathrm{T}}=\mu_{0} H_{\mathrm{c}}^{2} A$ ou pour notre application numérique

$$
P_{\mathrm{T}}=\left[3,9 \times 10^{-6} \frac{B_{\mathrm{s}}^{2}}{2 \mu_{0}}\right] A
$$

On peut alors citer pour mémoire la valeur $P_{\mathrm{T}}=0,08 \frac{B_{\mathrm{s}}^{2}}{2 \mu_{0}}$ atteinte dans les plus gros alternateurs de centrale. On trouvera dans les figures 7 les valeurs de $A$ obtenue en réluctance variable. Le rapport correspondant $\frac{P_{\mathrm{T}}}{B_{\mathrm{s}}^{2} / 2 \mu_{0}}$ est sensiblement plus faible que 0,08 , mais cette apparente infériorité doit être largement compensée par l'augmentation des surfaces d'entrefer spécifique de la structure à disques imbriqués.

L'étude de la fonction $A$, effectuée en fonction des dimensions des plots $(e, \lambda, s)$ et des ampères tours inducteurs $(\mathcal{V})$ est donc intrinsèquement celle de la pression magnétique tangentielle moyenne, elle est valable a priori pour n'importe quel type de machine à réluctance. Dans le cas particulier de la structure à disque, l'optimisation de $A$ conduit, de plus, naturellement à celle du couple volumique.

$$
C_{\mathrm{v}}=\frac{P_{\mathrm{v}}}{\Omega}=\mu_{0} H_{\mathrm{c}}^{2} \frac{R}{L} A .
$$

Le rapport de dimension $R / L$ conditionne en effet la stabilité transversale des disques et doit être choisi le plus élevé possible. Il peut donc être considéré comme un paramètre d'ordre mécanique et peu dépendant de $\lambda, e, s$ et $\vartheta$.

Signalons enfin que l'étude de la fonction $A$ s'applique aisément au cas où la fréquence $f$ d'alimentation de la machine est imposée. On a en effet dans ce cas une puissance volumique $P=\mu_{0} H_{\mathrm{c}}^{2} f \lambda A$ et la transposition des résultats est aisée.

Remarque : L'étude paramétrique est orientée ici sur l'évaluation des puissances volumiques disponibles dans ce type de machine; elle diffère en ce sens d'autres travaux dans lesquels la grandeur étudiée est plutôt un rapport de perméances [6], [7], [8].

2.4 RÉSUltatS OBTENUS. - 2.4.1 Lignes de champ. - A partir du champ $\left[h_{x}, h_{y}\right]$, calculé à tout point du maillage, un programme annexe trace les lignes de champ équiflux. Les tracés sont obtenus en conjonction et en opposition pour une même différence de potentiel magnétique et avec le même nombre de ligne de champ. On présente, pour $\lambda=1,5, e=0,07$ et $s=0,42$ la conjonction en régime de basse et très haute saturation (Fig. $5 a$ et $5 b$ ). Idem pour l'opposition (Fig. $5 c$ et $5 d$ ).

2.4.2 Etude de la fonction caractéristique $A(\lambda, e$, $s$, $)$. - Pour obtenir $A$, il suffit de tracer les courbes $\mathscr{F} / \lambda^{2}$ en fonction de $\mathcal{U}$, pour la conjonction et pour 


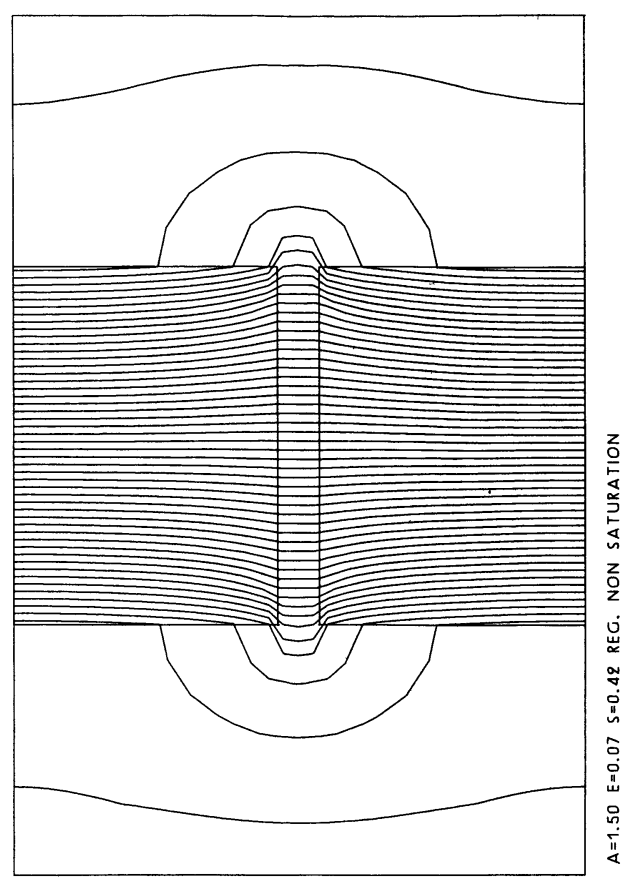

a)

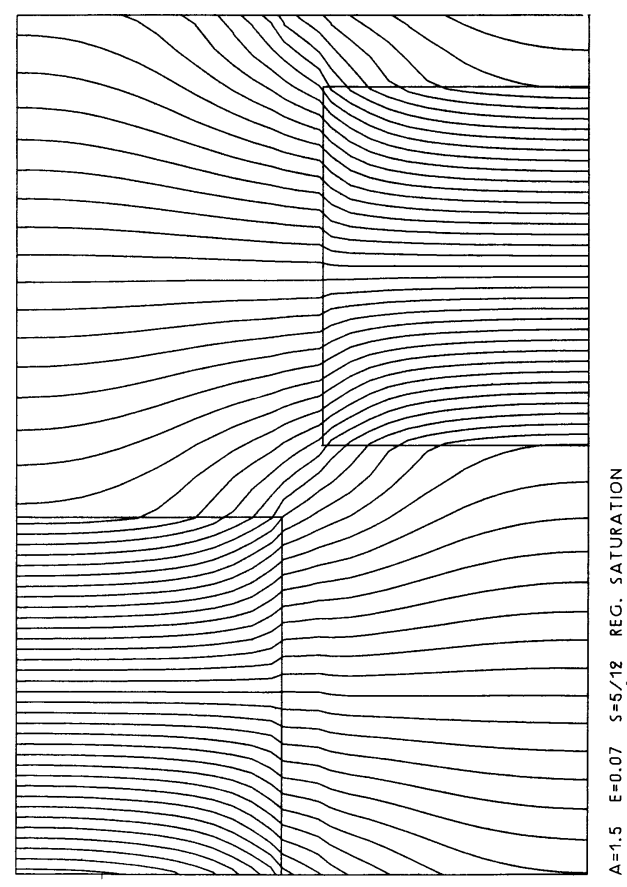

c)

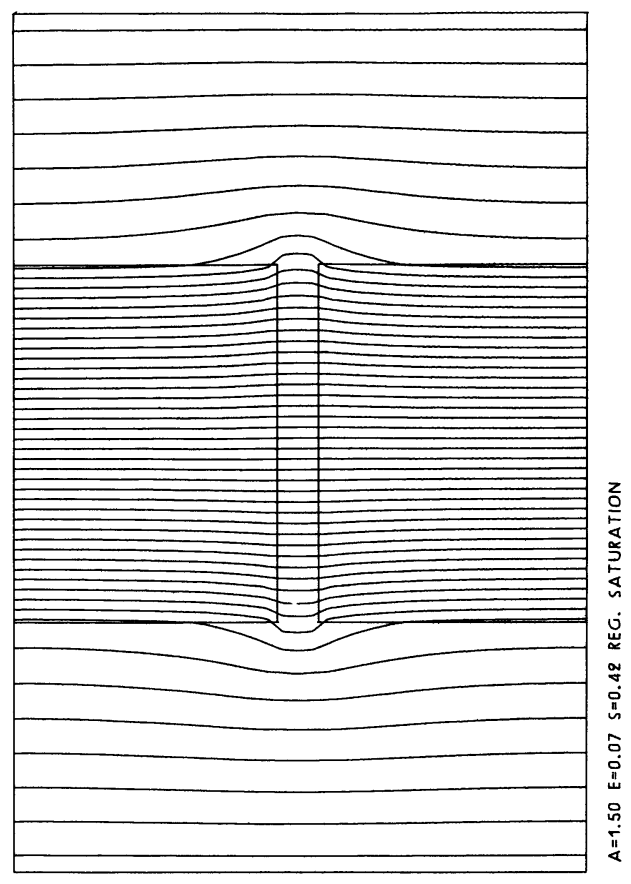

b)

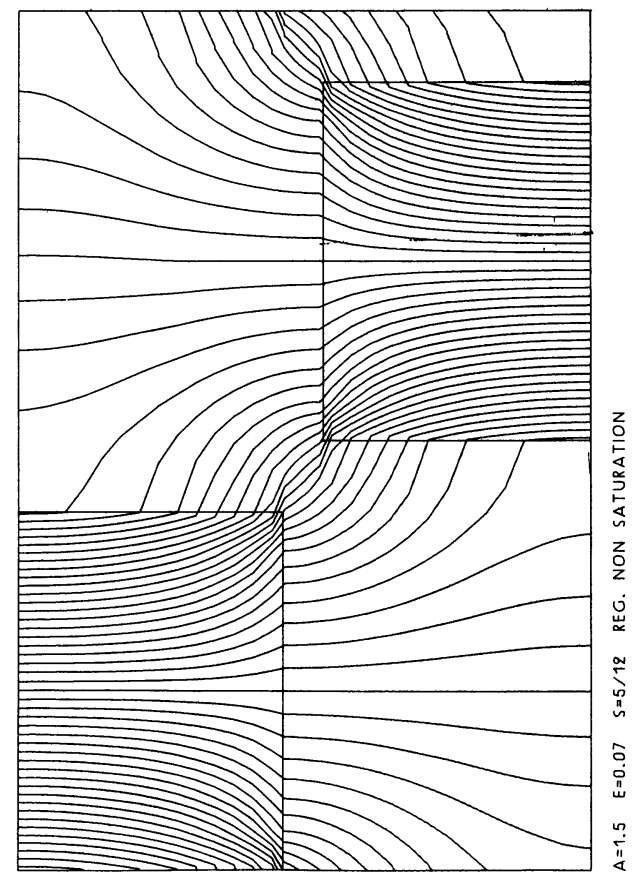

d)

Fig. $5(a, b, c, d)$. - Lignes de champ à différents régimes de satu ration.

[Field tubes at differents saturation regimes.] 

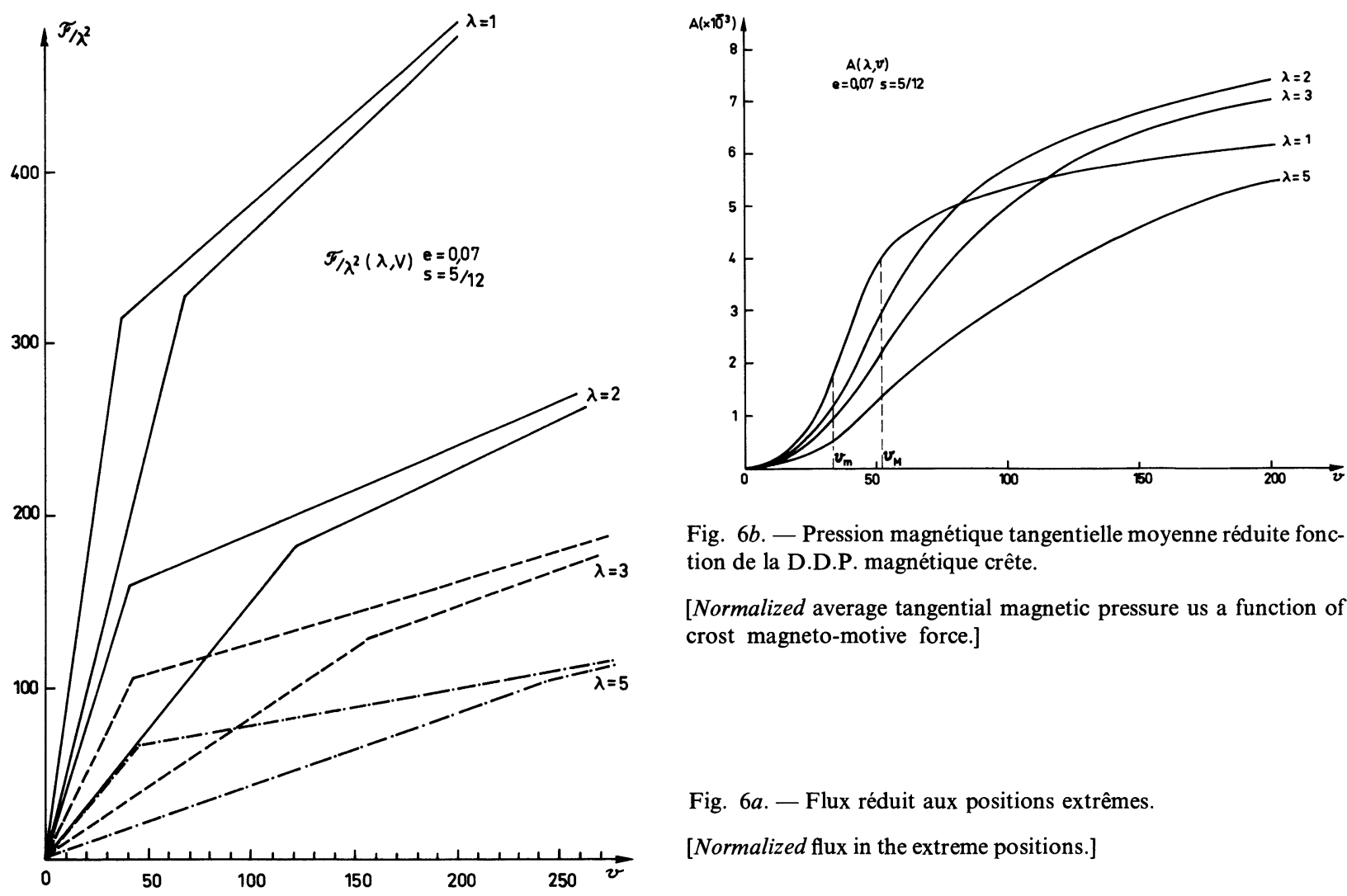

Fig. $6 b$. - Pression magnétique tangentielle moyenne réduite fonction de la D.D.P. magnétique crête.

[Normalized average tangential magnetic pressure us a function of crost magneto-motive force.]

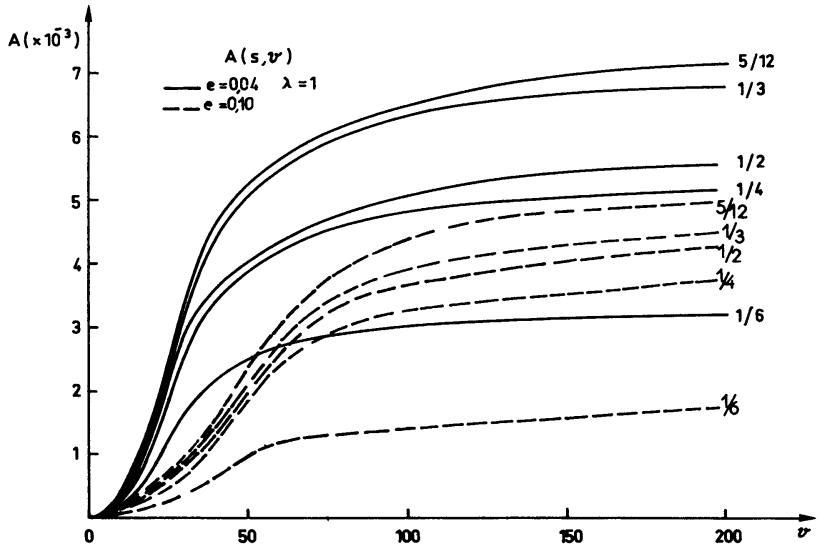

a)

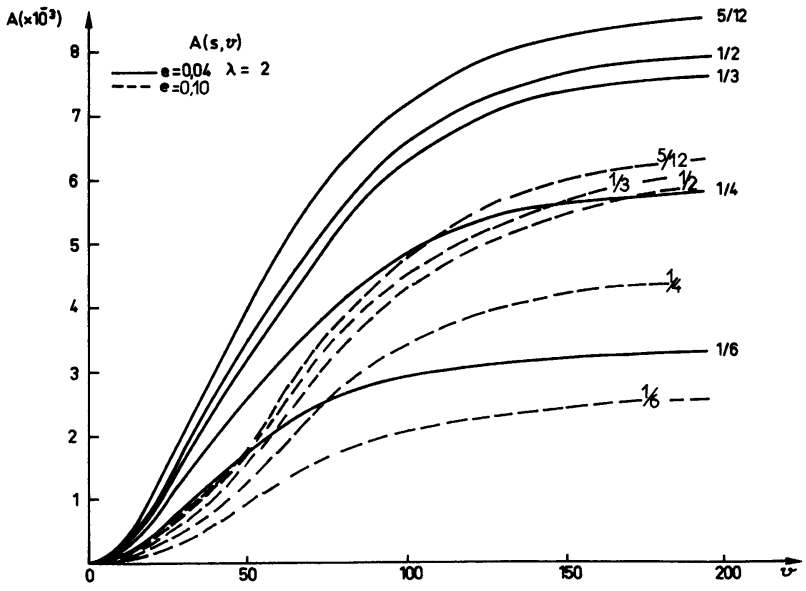

b)
Fig. $6 a$. - Flux réduit aux positions extrêmes.

[Normalized flux in the extreme positions.]

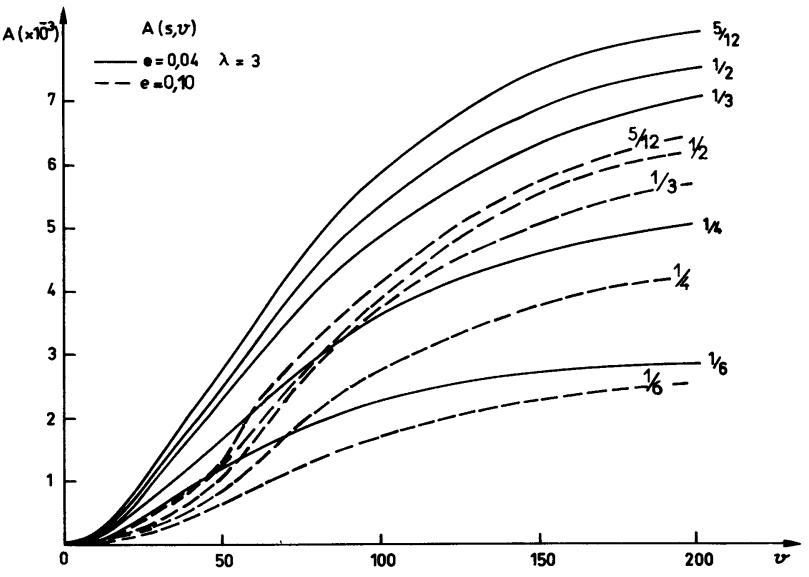

c)

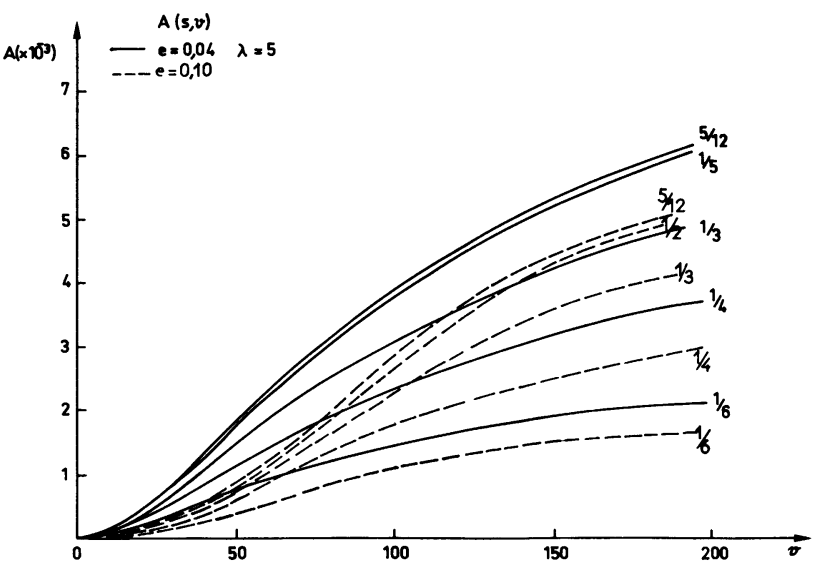

d) 
l'opposition. $A(\mathcal{U})$ est alors donné par l'aire comprise entre ces courbes et par la droite $v$ constante. Les figures $6 a$ et $6 b$ donnent un exemple de cette procédure pour $e=0,07, s=5 / 12$ et différentes valeurs de $\lambda$.

Pour déterminer complètement la fonction $A$, nous présentons les courbes $A(\mathcal{Y})$ sur les figures $7 a, 7 b, 7 c$ et $7 d$. Les lignes en traits pleins correspondent à $e=0,04$, tandis que celles en traits pointillés $e=0,10$. Une interpolation linéaire entre ces courbes donne, avec une bonne approximation, les valeurs intermédiaires de $e$.

En regardant ces résultats, on remarque que :

- sur les figures 7 , pour $e$ et $\lambda$ constants, $A$ est maximum lorsque $s$ est voisin de $5 / 12$ et ce, pour n'importe quelle valeur de $\mathcal{V}$.

- sur les figures 6 , avec $e$ et $s$ constants, il y a pour chaque valeur de $\vartheta$ une valeur de $\lambda$ correspondant au maximum de $A$ et qui, grosso-modo, augmente au fur et à mesure que $\vartheta$ augmente.

- à partir d'une certaine valeur de $\mathcal{V}$, on tombe dans une plage de travail qui présente peu d'intérêt car $A$ croît très lentement avec $v$.

L'examen de ces courbes montre également que l'entrefer relatif $e$ doit être le plus petit possible, les limites venant de contraintes mécaniques de construction. On note aussi que les ampères tours réduits $U$ doivent être aussi élevés que possible, la limitation venant cette fois des pertes joules admissibles dans le bobinage. On peut donc, considérer que, après un choix préalable de $e$ et $\mathcal{U}$, il faille adopter deux valeurs de $\lambda$ et $s$ maximisant $A$. Le problème est résolu sur les courbes des figures 8 . Celles-ci déduites des précédentes donnent :

sur la figure $8 a:$ la détermination de $\lambda$,

sur la figure $8 b:$ le choix résultant de $s$,

sur la figure $8 c$ : la valeur de $A$ obtenue.

3. Extension tridimensionnelle du modèle. - Nous signalons au début de ce travail l'intérêt que nous apportons aux machines à $R$.V. à disques imbriqués.

On peut distinguer deux structures différentes de disques, en ce qui concerne la conception des plots.

a) Type 1 : les disques à plot de section variable ayant un rapport fer-air $\frac{l_{2}}{l_{1}-l_{2}}$ indépendant du rayon (Fig. 9a).

b) Type 2 : les disques à plot de section constante ayant un rapport fer-air $\frac{l_{2}}{l_{1}-l_{2}}$ variable, le long du rayon (Fig. 9b).

Fig. $7(a, b, c, d)$. - Pression magnétique tangentielle moyenne réduite fonction de la D.D.P. et des paramètres de forme.

[Normalized average tangential magnetic pressure as a function of D.D.P. and design parameters.]

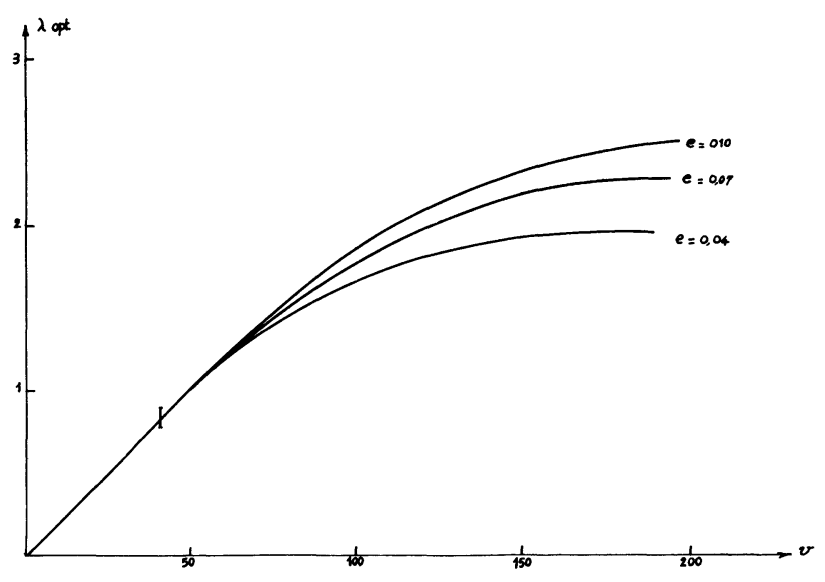

Fig. 8a. - Détermination optimale de $\lambda$.

[Optimal determination of $\lambda$.]

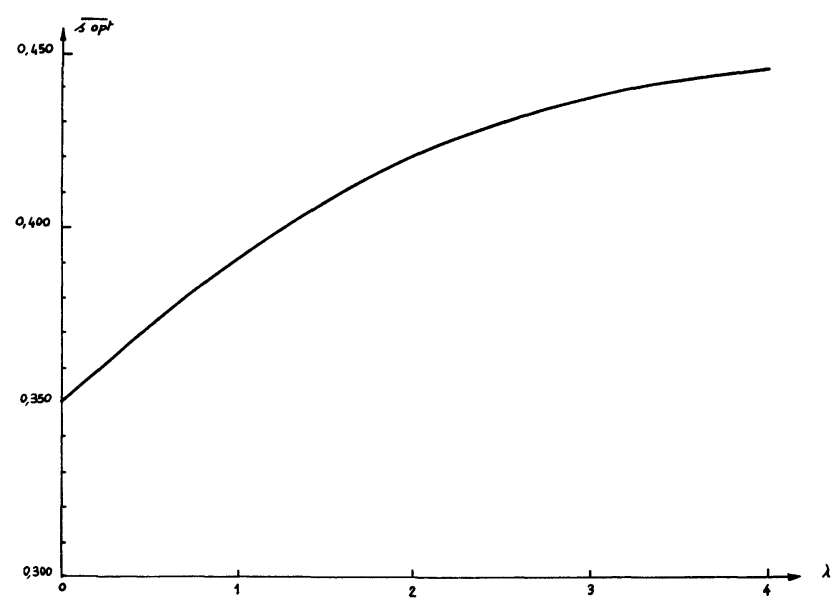

Fig. $8 b$. - Choix résultant de $s$.

[Resulting $s$.

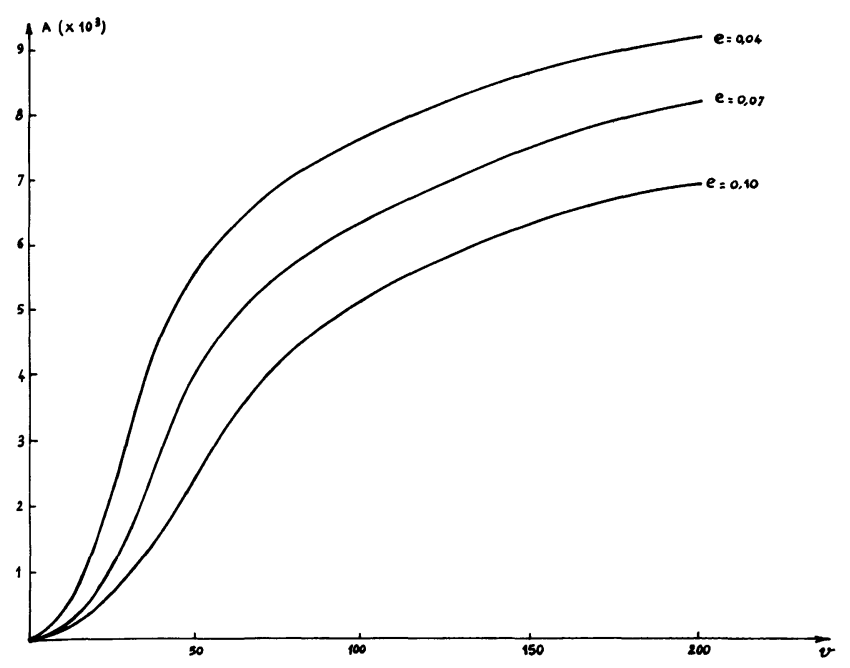

Fig. $8 c$. - Pression magnétique tangentielle optimale résultante.

[Resulting optimal tangential magnetic pressure.] 


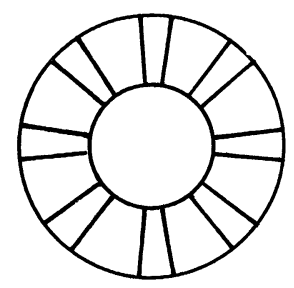

a)

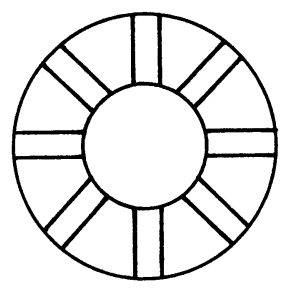

b)
Fig. $9(a$ et $b)$. - Section radiale des deux types principaux de plots. [Radial section of two principal types of teeth.]

Nous présentons sur la figure 10 un ensemble de plot-type 1 avec la partie d air correspondante

- le paramètre $\lambda=l_{1} / L$ est directement proportionnel au rayon,

- le paramètre $s=l_{2} / l_{1}$ est constant,

- le paramètre $e=E / L$ est constant.

Pour le plot-type 2, dans les mêmes conditions, on constate que :

$-\lambda$ est directement proportionnel au rayon,

- $e$ est maintenu constant,

$-s$ est inversement proportionnel au rayon.

Nous supposons choisis, a priori, $e$, $v$ et les rayons extérieur et intérieur des plots $R_{\mathrm{E}}$ et $R_{1}$; le choix des rayons définit l'encombrement de la machine. D'après ce que nous avons vu en 2.4.2 le choix de $e$ et $v$ définit sur les figures 8 une seule configuration optimale de plot.

Deux problèmes sont alors à résoudre :

- définir un rayon $R_{\mathrm{M}}$ entre $R_{\mathrm{E}}$ et $R_{1}$ sur lequel est adopté le paramètre $\lambda_{\text {opt }}$ de la figure $8 a$,

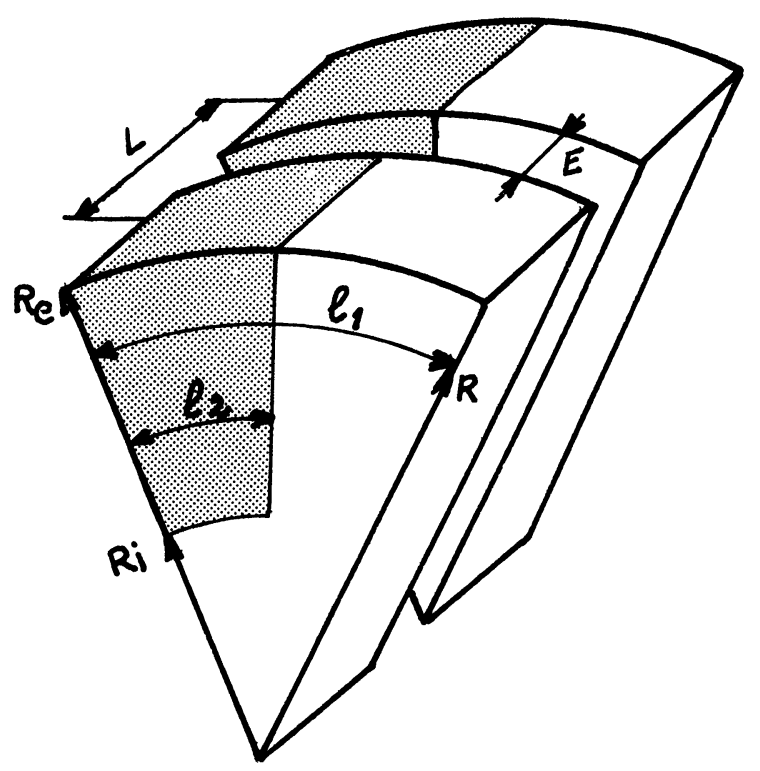

Fig. 10. - Perspective des plots du premier type.

[Perspective of first type of teeth.]
- chiffrer la perte de puissance résultant de la variation de $\lambda$ le long du rayon, pour les plots-type 1 et celle due aux variations de $\lambda$ et $s$ pour les plotstype 2 .

L'expression de la puissance volumique

$$
P_{\mathrm{v}}=\mu_{0} H_{\mathrm{c}}^{2} \frac{V}{L} A=\mu_{0} H_{\mathrm{c}}^{2} \frac{\Omega R}{L^{\prime}} A(R)
$$

donne pour une longueur axiale $L_{\mathrm{a}}$ de machine une puissance totale

$$
P=\int_{0}^{2 \pi} \int_{R_{\mathrm{I}}}^{R_{\mathrm{E}}} \mu_{0} H_{\mathrm{c}}^{2} \frac{\Omega R}{L} A(R)(R \mathrm{~d} \theta)\left(L_{\mathrm{a}} \mathrm{d} R\right)
$$

soit

$$
P=2 \pi \mu_{0} H_{\mathrm{c}}^{2} \frac{\Omega}{L} L_{\mathrm{a}} \int_{R_{\mathrm{I}}}^{R_{\mathrm{E}}} A(R) R^{2} \mathrm{~d} R
$$

La fonction $A(R)$, maximum et égale à $A_{\mathrm{M}}$ pour $R=R_{\mathrm{M}}$ est déductible des courbes des figures 7 . On a :

$$
A(R)=A\left(e, \lambda=\lambda_{\mathrm{opt}} \frac{R}{R_{\mathrm{M}}}, s, \vartheta\right)
$$

Supposons une machine fictive où tout le volume fonctionnerait avec la même puissance volumique $P_{\mathrm{VM}}=\mu_{0} H_{\mathrm{c}}^{2} \frac{\Omega R_{\mathrm{e}}}{L} A_{\mathrm{M}}$. Cette puissance $P_{\mathrm{VM}}$ correspond au maximum localement disponible puisque la valeur $\lambda=\lambda_{\text {opt }}(\vartheta, e)$ est placée à la périphérie, au rayon maximum $R=R_{\mathrm{E}}$.

Le rapport

$$
F=\frac{\int_{R_{\mathrm{I}}}^{R_{\mathrm{E}}} A(R) R^{2} \mathrm{~d} R}{0,5 R_{\mathrm{e}}\left(R_{\mathrm{e}}^{2}-R_{\mathrm{l}}^{2}\right) A_{\mathrm{M}}}
$$

sert à définir la réduction de performance obtenue sur la machine réelle relativement à celle fictive où tout le volume travaillerait à la puissance volumique $P_{\mathrm{vM}}$. Lorsque les ampères tours réduits $\vartheta$ et $e$ sont donnés, pour chaque valeur de $R_{\mathrm{E}} / R_{1}$ il existe une configuration optimale de disque. Celle-ci est définie par le quotient $R_{\mathrm{M}} / R_{\mathrm{E}}$ donnant une valeur maximum $F_{\mathrm{M}}$ au rapport $F$. Les calculs montrent que les rapports $R_{\mathrm{M}} / R_{\mathrm{E}}$ et $F_{\mathrm{M}}$ varient peu avec $\vartheta$ et $e$ et que l'on peut les exprimer en fonction de $R_{\mathrm{E}} / \boldsymbol{R}_{\mathbf{l}}$ (Fig. 11).

- A titre d'exemple d'utilisation de ces courbes, traitons le cas d'une machine telle que :

$$
\begin{gathered}
L=0,02 \mathrm{~m} ; \quad e=0,07 ; \quad \mathcal{U}=100 ; \\
R_{\mathrm{E}}=0,20 \mathrm{~m} ; \quad R_{\mathrm{I}}=0,10 \mathrm{~m} ; \\
V=\text { vitesse périphérique }=150 \mathrm{~m} / \mathrm{s} ; \\
B_{\text {sat }}=2 \mathrm{~T}\left(H_{\mathrm{c}}=2220 \mathrm{~A} / \mathrm{m}\right) .
\end{gathered}
$$




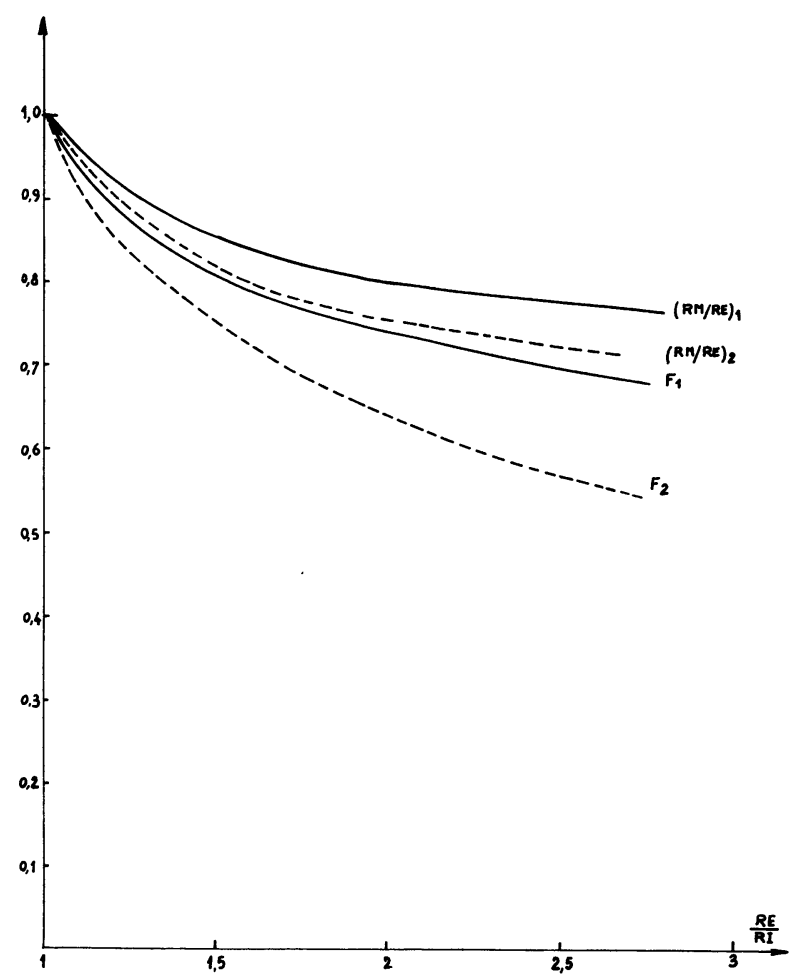

Fig. 11. - Localisation du rayon où sont placés les paramètres de forme optimaux.

[The radius at which the optimal design parameters should be respected.]

- Des figures 8 , avec $e=0,07$ et $v=100$, on obtient $\lambda_{\text {opt }}=1,73 ; s_{\text {opt }} \simeq 5 / 12$ et $A_{\mathrm{M}}=6,2 \times 10^{3}$.

- De la figure 11 , pour $R_{\mathrm{E}} / R_{\mathrm{I}}=2$ on déduit respectivement :

a) Pour la machine de type 1 :

$$
\frac{R_{\mathrm{M}}}{R_{\mathrm{E}}}=0,804 \quad \text { et } \quad F_{\mathrm{M}}=0,745
$$

avec $\lambda=1,73$ on a $l_{1}=1,73 \times 0,02=0,0346$; $R_{\mathrm{M}}=0,1608$. Le nombre de plots est donc

$$
N=\frac{2 \cdot \pi \cdot 0,1608}{0,0346} \simeq 29 \text {. }
$$

La puissance par unité de longueur de la machine est

$$
P=\mu_{0} H_{\mathrm{c}}^{2} \frac{V}{L} A_{\mathrm{M}} F_{\mathrm{M}} \pi\left(R_{\mathrm{c}}^{2}-R_{\mathrm{i}}^{2}\right)=2,02 \times 10^{7} \mathrm{~W} / \mathrm{m}
$$

b) Pour la machine de type 2 :

$$
\begin{gathered}
\frac{R_{\mathrm{M}}}{R_{\mathrm{E}}}=0,75 ; \quad F_{\mathrm{M}}=0,640 \\
N=24 \text { plots et } P=1,74 \times 10^{7} \mathrm{~W} / \mathrm{m} .
\end{gathered}
$$

Les ampères-tours par unité de longueur de machine, pour les deux types de disques sont :

$$
I=H_{\mathrm{c}} v=2,22 \times 10^{5} \mathrm{~A} \cdot \mathrm{t} / \mathrm{m} .
$$

Conclusion. - Rappelons en conclusion que l'optimisation intrinsèque effectuée ne tient compte que de la région interne où sont placés les plots.

Dans les réalisations pratiques, les performances globales obtenues seront d'autant plus proches de celles exposées que l'influence des régions externes sera plus faible.

En outre, d'autres notions importantes comme celle du rendement ou du facteur de puissance nécessitent naturellement une étude complémentaire où interviendrait l'ensemble convertisseur statique-machine.

Dégageons enfin les premières vérifications expérimentales effectuées. Celles-ci, données à titre indicatif, ne remettent pas en cause l'optimisation de forme exposée. Les vérifications ont été faites sur un prototype comportant un disque mobile entouré de deux disques fixes; sa conception originale, facilite l'évaluation directe des différences de potentiel et des flux intervenant dans la seule région des plots [10]. Si la mesure des flux est aisée, celle des différences de potentiel est par contre plus délicate, il est en effet difficile d'éliminer complètement l'influence du circuit de retour. On a pu, néanmoins relever une erreur relative inférieure à $10 \%$ sur le calcul des flux en conjonction et opposition à différentes D.D.P. magnétiques et à $25 \%$ en ce qui concerne leur écart.

Dans une première étape, et en absence de modèle numérique tridimensionnel, on peut donc considérer que l'approximation bidimensionnelle et l'extension présentée sont satisfaisantes. 


\section{Bibliographie}

[1] Théorie des machines comportant des matériaux ferromagnétiques. Rioux, C., publication interne LF no 70, sept. 1977, bât. 214, 91405 Orsay.

[2] Finite Element Solution of Saturable Magnetic Field Problems. Silvester, P., Chari, M. V. K., IEEE Trans. Power Appar. Syst. PAS 89 (1970) No 7.

[3] Analyse numérique du champ magnétique d'un alternateur par éléments finis. Glow INSKI, R. et MARROCO, A., Computer Methods appl. Mech. Eng. 3 (1974) 55-85.

[4] Modélisation des machines tournantes et prédétermination de leurs caractéristiques. SABONNADIÈRE, J. C. et al., Rev. Gén. Electr. 84 (1975) No 9.

[5] Comparaison des diverses modélisations numériques d'un système magnétique saturable. JABLON, C., TOURNAIRE, M., Rev. Gén. Electr. 84 (1975) № 9.
[6] Magnetic Permeance of Identical Double Slotting. MUKERJI, K. C. et Neville, S., Proc. IEE 118 (1979) No 9.

[7] Vernier Reluctance Motor. Mukeru, K. C., Tustin, A., Proc. IEE 121 (1974) No 9.

[8] Optimal Design of a variable Reluctance Stepping Motor. Athany, V. V. and Khurana, S. K., International Conference on electrical Machines, sept. 11, 12, 13, 1978, Congressenpaleis Brussels, Belgium.

[9] Théorie générale comparative des machines électriques établie à partir des équations du champ électromagnétique. RIoux, C., Rev. Gén. Electr. 79 (1970) 415.

[10] Contribution to the study of an interleaved disk reluctance machine. GOYET, R., RIOUx, C., International conference on electrical machines. September 11, 12, 13, 1978. Brussels, Belgium. 